\title{
Human immune system diversity and its implications in diseases
}

Journal of Human Genetics (2015) 60, 655-656; doi:10.1038/jhg.2015.101

A part from a small number of single-gene diseases, the majority of autoimmune or immune-related diseases are complex diseases, where a combination of multiple genetic and non-genetic factors is thought to play a role. The diversity of human immune system genes, arguably shaped under the selection pressure of microorganisms, is critically involved in the genetic background of such diseases. In the planning of this review issue, we intended to incorporate a viewpoint from population genetics into the genetics of immune-related diseases. As a result, this issue turned out to be a unique collection of stimulating reviews encompassing human genome science, immunogenetics, clinical immunology and anthropology.

Genome-wide association studies somewhat unexpectedly revealed that the alleles strongly associated with susceptibility to various autoimmune or immune-related disorders are often shared by multiple diseases, including those with little clinical resemblance to each other. $\mathrm{Wu}$ et al. ${ }^{1}$ provide an excellent overview of genome-wide association studies and Immunochip studies, and present a picture of the 'genomic landscape' of immune-related diseases in general. They also give a detailed discussion of the loci associated with multiple diseases, including major histocompatibility complex (MHC), the IL-12 family, the Th17 pathway and the tumor necrosis factor (TNF) superfamily.

Ramos et al. demonstrate how profoundly natural selection has influenced the diversity of immune system genes among human populations. In addition, they provide a comprehensive list of the autoimmune disease-associated alleles across the genome that may be under natural selection, which is highly informative and valuable. ${ }^{2}$

Nakaoka et al. focus on the HLA haplotypes in the Japanese population, and discuss how they are related to, and also different from, those in the East and Southeast Asian populations. Moreover, they show regional differences in HLA haplotype distribution among the Japanese population. Finally, they discuss the relevance of the gene flow from Neanderthals and Denisovans in shaping the HLA haplotypes in present-day Asians. ${ }^{3}$

Switching the channel to the clinical aspects, $H L A$, or the $M H C$ region, remains the strongest genetic risk factor for the majority of immune system disorders. However, after over 40 years since the discovery of the remarkable association between ankylosing spondylitis and HLA-B27-arguably the first striking association between HLA and disease- the molecular mechanism of HLA association remains unclear. Furukawa et al. provide an excellent update on the HLA association with rheumatoid arthritis (RA). They not only discuss the $H L A$ association with susceptibility to overall RA, but also with clinical subsets, complications and adverse reactions against anti-rheumatic drugs. Such information is highly relevant in the setting of personalized medicine for RA. ${ }^{4}$

Miyadera et al. focus on molecular mechanisms that might account for the HLA-class II association with type 1 diabetes and RA. Here, based on their own observations, they propose a 'MHC stability model' which hypothesizes that the risk $D Q A 1-D Q B 1$ haplotype may be associated with type 1 diabetes through the production of unstable HLA-DQ molecules. ${ }^{5}$ Further studies are needed to validate this fascinating hypothesis.

One of the striking characteristics of human immune system genes is the importance of multigene families. The immunoglobulin (Ig)-like receptor family is one such example. Ig-like receptor genes such as Fcy receptor (FCGR) genes and killer-cell Ig-like receptor $(K I R)$ genes have been associated with a number of diseases. Here, Hirayasu et al. ${ }^{6}$ review another intriguing group of Ig-like receptor genes, leukocyte Iglike receptor (LILR) genes. LILR is a family composed of 11 functional genes (activating, inhibitory and soluble) and 2 pseudogenes. Although their biological functions are just beginning to emerge, some members of the LILR family work as receptors of HLA class I, and LILRA4 is expressed in the plasmacytoid dendritic cells and functions as an inhibitory receptor. Thus, the LILR family has an important role in the immune system, and in fact, a number of disease associations, including various autoimmune diseases and prostate cancer, have already been reported.

Finally, Hosomichi et al. discuss exciting progress on $M H C$ research using next-generation sequencing (NGS). ${ }^{7}$ NGS can overcome a number of previous limitations in $M H C$ research; for example, conventional genotyping methods could only 'estimate' the alleles of an individual because of multiple heterozygous polymorphic positions. Using NGS, one can experimentally determine the 'phase' of the haplotype. The authors further emphasize the importance of regulatory region polymorphisms, which can affect the expression of $H L A$. Little is currently known with respect to the differences in the expression levels of individual HLA alleles. Thus, NGS analysis of MHC region will open a new era of 'HLA-omics'.

We sincerely thank the contributors and editorial staff who have made this unique review issue possible. We hope that the readers enjoy this issue, and we will be thrilled if this small collection of stimulating reviews is able to pave the way to close and fruitful collaboration between immunogenetics and population genetics.

Naoyuki Tsuchiya ${ }^{1}$ and Jun Ohashi ${ }^{2}$ ${ }^{1}$ Molecular and Genetic Epidemiology Laboratory, Faculty of Medicine, University of Tsukuba, Tsukuba, Japan and 
${ }^{2}$ Department of Biological Sciences, Graduate School of Science, The University of Tokyo, Tokyo, Japan E-mail: tsuchiya-tky@umin.net

$1 \mathrm{Wu}, \mathrm{X} ., \mathrm{Chen}, \mathrm{H}$. \& Xu, H. The genomic landscape of human immune-mediated diseases. J. Hum. Genet. 60, 675-681 (2015).

2 Ramos, P. S., Shedlock, A. M. \& Langefeld, C. D. Genetics of autoimmune diseases: insights from population genetics. J. Hum. Genet. 60, 657-664 (2015).
3 Nakaoka, H. \& Inoue, I. Distribution of HLA haplotypes across Japanese Archipelago: similarity, difference and admixture. J. Hum. Genet. 60, 683-690 (2015).

4 Furukawa, H. Oka, S., Shimada, K., Hashimoto, A. \& Tohma, S. Human leukocyte antigen polymorphisms and personalized medicine for rheumatoid arthritis. J. Hum. Genet. 60, 691-696 (2015)

5 Miyadera, H. \& Tokunaga, K. Associations of human leukocyte antigens with autoimmune diseases: challenges in identifying the mechanism. J. Hum. Genet. 60 697-702 (2015)

6 Hirayasu, K. \& Arase, H. Functional and genetic diversity of leukocyte immunoglobulinlike receptor and implication for disease associations. J. Hum. Genet. 60, 703-708 (2015)

7 Hosomichi, K., Shiina, T., Tajima, A. \& Inoue, I. The impact of next-generation sequencing technologies on HLA research. J. Hum. Genet. 60, 665-673 (2015). 